\title{
Singular Hamiltonians in models with spontaneous Lorentz symmetry breaking
}

\author{
Michael D. Seifert \\ Department of Physics, Astronomy, and Geophysics, Connecticut College, \\ 270 Mohegan Avenue, New London, Connecticut 06320, USA
}

(Received 15 May 2019; published 23 September 2019)

\begin{abstract}
Many current models which "violate Lorentz symmetry" do so via a vector or tensor field which takes on a vacuum expectation value, thereby spontaneously breaking the underlying Lorentz symmetry of the Lagrangian. One common way to construct such a model is to posit a smooth potential for this field; the natural low-energy solution of such a model would then be excepted to have the tensor field near the minimum of its potential. It is shown in this work that some such models, while appearing well posed at the level of the Lagrangian, have a Hamiltonian which is singular on the vacuum manifold and are therefore ill posed. I illustrate this pathology for an antisymmetric rank-2 tensor field and find sufficient conditions under which this pathology occurs for more general field theories.
\end{abstract}

DOI: 10.1103/PhysRevD.100.065017

\section{INTRODUCTION}

The prospect of finding new physics via Lorentz symmetry violation has been of significant interest over the past couple of decades. In many such models, Lorentz symmetry is broken spontaneously; one postulates the existence of a new fundamental field that is not a Lorentz scalar and assigns dynamics to this field that obey Lorentz symmetry but lead it to take on a nonzero "vacuum expectation value." The existence of this nonzero Lorentz vector or tensor field then provides a preferred geometric structure in spacetime.

In effect, such a field would provide a "hook" upon which one can "hang" frame-dependent effects. In the presence of a Yukawa-like couplings between this new field and conventional matter fields, the results of experiments would depend on the relative orientation in spacetime of the observer's 4-velocity and the new field, leading to framedependent effects. Such a field is frequently called a "Lorentz-violating" field, though this is something of a misnomer; the postulated field would still transform between frames via the standard Lorentz transformation laws. The dynamics of such fields in flat spacetime have been studied in their own right [1-4] and similar models have also been developed in the context of curved spacetime as possible modifications to general relativity [5-7].

\footnotetext{
*mseifer1@ conncoll.edu
}

Published by the American Physical Society under the terms of the Creative Commons Attribution 4.0 International license. Further distribution of this work must maintain attribution to the author(s) and the published article's title, journal citation, and DOI. Funded by SCOAP.
Many (though not all) of the above-cited models share two features. First, they accomplish the spontaneous breaking of Lorentz symmetry by assigning a potential energy $V\left(\Psi^{\cdots}\right)$ to some Lorentz tensor $\Psi^{\cdots}$. This potential is constructed in a "Higgs-like" way, with a set of minima forming a vacuum manifold in field space. Since the field $\Psi^{\cdots}$ is a Lorentz tensor but we want the underlying equations of motion to obey Lorentz symmetry, we have to construct the potential out of one or more Lorentz scalars that are dependent on $\Psi^{\cdots}$. The vacuum manifold will be determined by a set of conditions on these Lorentz scalars. For example, if we wish to construct a model in which a Lorentz vector field $A^{a}$ spontaneously breaks Lorentz symmetry, it is not hard to see that the potential $V\left(A^{a}\right)$ must be some function of the 4-vector norm $A_{a} A^{a}$, and the vacuum manifold will be the set of all 4-vectors of a particular norm.

Second, the kinetic terms in the Lagrangians for these theories must be constructed with care. Assuming that the underlying Lagrangian is second order and gives rise to second-order equations of motion, the kinetic terms will typically involve a contraction of the spacetime derivative $\nabla_{a} \Psi_{b_{1} \cdots b_{n}}$ with itself. However, one cannot usually simply write down a kinetic term of the form $\nabla_{a} \Psi_{b_{1} \cdots b_{n}} \nabla^{a} \Psi^{b_{1} \cdots b_{n}}$ and call it a day. Since the Minkowski metric is indefinite, such a kinetic term will lead to terms with the "wrong sign" of the kinetic energy in the Hamiltonian, which will generically lead to instabilities in the classical solutions. Instead, the kinetic term is usually constructed out of more complicated combinations of the field derivatives, such that the problematic terms in the kinetic energy do not appear (via cancellations between various contractions). Again, 
returning to the case of a 4-vector field for illustration, it is not hard to see that a kinetic term of the form $\frac{1}{2} \nabla_{a} A_{b} \nabla^{a} A^{b}$ will include terms of the form $\frac{1}{2}\left[\dot{A}_{0}^{2}-\dot{\vec{A}}^{2}\right]$, which is indefinite. However, the time derivatives that arise from "Maxwell" kinetic term $-\frac{1}{4} F_{a b} F^{a b}$ (with $F_{a b}=2 \nabla_{[a} A_{b]}$ ) are all of the same sign, evading this problem.

The price one pays for this good behavior, however, is that the time derivatives of some fields do not appear at all in the Lagrangian. This leads to the model being constrained; certain canonical field momenta must vanish identically, and so one cannot freely specify both the values of the fields and their time derivatives at some initial moment $t_{0}$.

The purpose of this work is to illustrate and explore a potential incompatibility between these two features. Specifically, a model which contains a constrained field with a vacuum manifold, while appearing well posed at the level of the Lagrangian, may in fact have a Hamiltonian that becomes singular on the vacuum manifold. This raises serious doubts about the viability of such a theory; it predicts that the field, if perturbed slightly from its vacuum manifold, could not smoothly evolve back to the vacuum manifold. This conflict does not occur in all Lorentzviolating field models but instead seems to arise when the "number of constraints" exceeds the codimension of the vacuum manifold in field space.

The work is structured as follows. A brief summary of the techniques of Hamiltonian field theory pertinent to this result is presented in Sec. II. Section III contains an illustration of how a singular Hamiltonian can arise in a Lorentz-violating field theory, by examining the dynamics of a rank-2 antisymmetric tensor field $B_{a b}$ in a Lagrangian that is designed to spontaneously break Lorentz symmetry. Finally, Sec. IV discusses how this pathology could arise in a general field theory.

Throughout this work, I will use units in which $\hbar=$ $c=1$; the metric signature will be $(-+++)$. Roman indices $a, b, c, \ldots$ will be used to denote spacetime tensor indices; $i, j, k, \ldots$ will be used to denote spatial indices, where necessary. Greek indices $\alpha, \beta, \gamma, \ldots$ will generally only be used to denote indices in field space. All expressions involving repeated indices (either tensor indices or field-space indices) can be assumed to obey the Einstein summation convention, unless explicitly stated otherwise; in particular, for a significant fraction of Sec. IV B and in the Appendix, the field-space summations will be written out explicitly. While I will be working exclusively in the realm of flat spacetime, I will still use the symbol $\nabla_{a}$ (or $\nabla_{i}$ ) to denote spacetime (or spatial) derivatives; the symbol $\partial$ will be reserved for partial derivatives of functions of fields (such as the field potential energy or the Lagrangian density) with respect to their arguments. The symbol $\delta$ will generally denote either variations of functionals or functional derivatives.

\section{HAMILTONIAN FIELD THEORY}

In classical mechanics, the construction of a Hamiltonian from a Lagrangian $L\left(q_{\alpha}, \dot{q}_{\alpha}\right)$ is a relatively straightforward process. One performs a Legendre transform on the Lagrangian, by defining the conjugate momenta $p_{\alpha}=$ $\partial L / \partial \dot{q}_{\alpha}$; inverting these relationships to write the velocities $\dot{q}_{\alpha}$ in terms of the coordinates $q_{\alpha}$ and the momenta $p_{\alpha}$; and finally writing the Hamiltonian as $H\left(q_{\alpha}, p_{\alpha}\right)=$ $p_{\alpha} \dot{q}_{\alpha}-L\left(q_{\alpha}, \dot{q}_{\alpha}\right)$, now viewing $\dot{q}_{\alpha}$ as a function of $q_{\alpha}$ and $p_{\alpha}$. One can then find the evolution of the coordinates and momenta via Hamilton's equations, or via the Poisson bracket,

$$
\frac{d f}{d t}=\{f, H\}
$$

where for any quantities $f$ and $g$ we have

$$
\{f, g\} \equiv \sum_{\alpha} \frac{\partial f}{\partial q_{\alpha}} \frac{\partial g}{\partial p_{\alpha}}-\frac{\partial g}{\partial q_{\alpha}} \frac{\partial f}{\partial p_{\alpha}} .
$$

In field theory for some set of fields $\psi_{\alpha}$, one would like to follow the same procedure, starting from a Lagrange density $\mathcal{L}\left(\psi_{\alpha}, \dot{\psi}_{\alpha}, \nabla_{i} \psi_{\alpha}\right)$. However, an important difference can arise when one attempts to perform the Legendre transform. It can happen that, due to the structure of the kinetic terms, the field velocities cannot all be written in terms of the field momenta. In general, this implies that one or more of the equations of motion for the field are actually constraint equations, not evolution equations.

One can, however, still attempt to construct a Hamiltonian that generates the time evolution of the system [in the sense of (1)] via a construction due to Dirac and Bergmann [8]. A brief description of the method can also be found in a paper by Isenberg and Nester [9], and the notation used here will largely follow the notation in that work. The construction proceeds as follows:

(i) Define the field momenta via the natural generalization:

$$
\pi_{\alpha}=\frac{\partial \mathcal{L}}{\partial \dot{\psi}_{\alpha}} .
$$

One can then attempt to invert these relationships to find $\dot{\psi}_{\alpha}$ as a function of $\pi_{\alpha}, \psi_{\alpha}$, and their derivatives. However, it may occur that certain equations (or combinations of equations) in (3) do not contain any velocities. These equations must be thought of as constraining the initial data and can be written in the form

$$
\Phi_{I}\left(\psi_{\alpha}, \vec{\nabla} \psi_{\alpha}, \pi_{\alpha}\right)=0
$$

for $I=1,2, \ldots, M$. The functions $\Phi_{I}$ are known as the primary constraints. 
(ii) Construct the base Hamiltonian density via a Legendre transform on the Lagrange density:

$$
\mathcal{H}_{0} \equiv \pi_{\alpha} \dot{\psi}^{\alpha}-\mathcal{L}
$$

It can be shown that all of the velocities $\dot{\psi}^{\alpha}$ will vanish in this process. However, the evolution generated by this base Hamiltonian $H_{0}=\int d^{3} x \mathcal{H}_{0}$ will not, in general, preserve the constraints (4). To obtain a Hamiltonian which preserves the constraints, one must "augment" the base Hamiltonian density by adding the constraints to it, each multiplied by an as-yet-undetermined Lagrange multiplier $u_{I}$ :

$$
\mathcal{H}_{A} \equiv \mathcal{H}_{0}+u_{I} \Phi_{I}
$$

This latter quantity is the augmented Hamiltonian density.

(iii) If the constraints are to be preserved by the augmented Hamiltonian $H_{A}=\int d^{3} x \mathcal{H}_{A}$, it must be the case that $\left\{\Phi_{I}, H_{A}\right\}=0$ for each constraint. If this Poisson bracket does not vanish identically, this will yield a secondary constraint $\Psi_{I}=0$, which the initial data must also obey. Similarly, this secondary constraint must also be conserved, so we demand $\left\{\Psi_{I}, H_{A}\right\}=0$ as well; this will then generate further secondary constraints, which must in turn be preserved, and so forth. The requirement that all of the constraints so generated be preserved may determine some or all of the hitherto undetermined Lagrange multipliers, in which case we can replace them in the augmented Hamiltonian with an expression written solely in terms of the fields and the momenta. It is also conceivable that we may find an inconsistent model (i.e., one of the constraint equations cannot be preserved under the time evolution generated by $H_{A}$ ).

When the dust settles, one is left with a Hamiltonian $H_{A}$ which generates the time evolution of the fields. We can use this Hamiltonian to count (in a simplified way) the number of degrees of freedom (d.o.f.) of the system; specifically,

$$
\begin{aligned}
N_{\text {dof }}= & \frac{1}{2}\left[\left(\begin{array}{c}
\text { number of } \\
\text { fields }
\end{array}\right)+\left(\begin{array}{c}
\text { number of } \\
\text { momenta }
\end{array}\right)\right. \\
& \left.-\left(\begin{array}{c}
\text { number of } \\
\text { constraints }
\end{array}\right)-\left(\begin{array}{c}
\text { number of undetermined } \\
\text { Lagrange multipliers }
\end{array}\right)\right] .
\end{aligned}
$$

The undetermined Lagrange multipliers that remain after this process are associated with gauge d.o.f. and so are unphysical. In the present work, however, the models under consideration will have all of their Lagrange multipliers determined, and (since the number of fields and momenta will be the same) we will have

$$
N_{\text {dof }}=\left(\begin{array}{c}
\text { no. of } \\
\text { fields }
\end{array}\right)-\frac{1}{2}\left(\begin{array}{c}
\text { no. of } \\
\text { constraints }
\end{array}\right) .
$$

\section{ANTISYMMETRIC RANK-2 TENSOR}

\section{A. Action}

To illustrate the problems which can arise in a model containing both constraints and a vacuum manifold, we consider the case of a rank-2 antisymmetric tensor field $B_{a b}=B_{[a b]}$. In four-dimensional spacetime, there are two possible invariants that can be constructed from this field, which we will denote as $X$ and $Y$,

$$
X=B^{a b} B_{a b} \quad Y=\mathfrak{B}^{a b} B_{a b},
$$

where

$$
\mathfrak{B}^{a b} \equiv \frac{1}{2} \epsilon^{a b c d} B_{c d}
$$

(Note that $\mathfrak{B}^{a b} \boldsymbol{B}_{a b}=-X$.) We consider an action of the form

$$
S=\int d^{4} x\left[-\frac{1}{12} F_{a b c} F^{a b c}-V(X, Y)\right],
$$

where

$$
F_{a b c} \equiv 3 \partial_{[a} B_{b c]}
$$

The Euler-Lagrange equations derived from this action will then be

$$
\frac{1}{2} \partial_{c} F^{c a b}-V_{X} B^{a b}-V_{Y} \mathfrak{B}^{a b}=0,
$$

where $V_{X} \equiv \partial V / \partial X$ and $V_{Y} \equiv \partial V / \partial Y$.

Since we will be attempting to construct a Hamiltonian for this model, we will need to perform a $3+1$ decomposition. Given a choice of time coordinate $t=x^{0}$ on our spacetime, we can decompose the field $B_{a b}$ into spatial vectors $\vec{P}$ and $\vec{Q}$, corresponding to its "electric" and "magnetic" parts respectively,

$$
P^{i}=B^{0 i} \quad Q^{i}=\frac{1}{2} \epsilon^{i j k} B^{j k},
$$

where $\epsilon^{i j k}$ is the volume element on a constant- $t$ hypersurface in spacetime. In terms of these, the kinetic term in the Lagrangians above can be rewritten as 


$$
-\frac{1}{12} F_{a b c} F^{a b c}=\frac{1}{2}\left[(\dot{\vec{Q}}-\vec{\nabla} \times \vec{P})^{2}-(\vec{\nabla} \cdot \vec{Q})^{2}\right],
$$

while the invariants $X$ and $Y$ become

$$
X=-2 \vec{P}^{2}+2 \vec{Q}^{2}, \quad Y=-4 \vec{P} \cdot \vec{Q} .
$$

For the sake of concreteness in what follows, we will want to have an explicit form for the potential $V$. To support Lorentz violation, the tensor $B_{a b}$ must have a nonzero expectation value which can couple to other matter fields. We therefore want to construct a potential such that there exist solutions to (13) where $B_{a b}$ is nonzero but constant. A potential $V$ which is linear in the invariants $X$ and $Y$ will lead only to solutions where $B_{a b}=0$, and we must therefore construct a potential which is quadratic in the invariants (and hence quartic in the fields),

$V\left(B_{a b}\right)=\frac{1}{2} \kappa_{1} X^{2}+\kappa_{2} X Y+\frac{1}{2} \kappa_{3} Y^{2}+\lambda_{1} X+\lambda_{2} Y$,

where the $\kappa_{i}$ and $\lambda_{i}$ coefficients determine the "shape" of the potential.

Equation (13) will then be satisfied for a constant tensor field if and only if $V_{X}=V_{Y}=0$, or ${ }^{1}$

$$
\left[\begin{array}{ll}
\kappa_{1} & \kappa_{2} \\
\kappa_{2} & \kappa_{3}
\end{array}\right]\left[\begin{array}{l}
X \\
Y
\end{array}\right]+\left[\begin{array}{l}
\lambda_{1} \\
\lambda_{2}
\end{array}\right]=0
$$

Assuming that $\kappa_{1} \kappa_{3}-\kappa_{2}^{2} \neq 0$, the solutions to (18) will be those where $X$ and $Y$ both have a particular value determined by the $\kappa_{i}$ and $\lambda_{i}$ coefficients. This solution space is the vacuum manifold of our model; it will be a four-dimensional manifold in the six-dimensional field space. $^{2}$ More generally, the dimension of the vacuum manifold will be 4 plus the dimension of the solution space of (18). For example, for the antisymmetric tensor models discussed in Refs. [10,11], the invariant $Y$ is undetermined, and thus the vacuum manifold is five dimensional.

\section{B. Constructing the Hamiltonian}

From the kinetic term in (15), we can find the conjugate momenta for the fields $\vec{P}$ and $\vec{Q}$, the former of which can be seen to vanish:

$$
\vec{\Pi}_{P}=\frac{\delta \mathcal{L}}{\delta \dot{\vec{P}}}=0, \quad \vec{\Pi}_{Q}=\frac{\delta \mathcal{L}}{\delta \dot{\vec{Q}}}=\dot{\vec{Q}}-\vec{\nabla} \times \vec{P}
$$

\footnotetext{
${ }^{1}$ The "if" part of this statement is obvious. To see the "only if" part, suppose that $B_{a b} \neq 0$ and $\alpha B_{a b}+\beta \mathfrak{B}_{a b}=0$ for some $\alpha, \beta \in \mathbb{R}$. Contracting this equation with $\epsilon^{a b c d}$ yields $\alpha \mathfrak{B}_{a b}-\beta B_{a b}=0$, and these two equations together imply that $\alpha=\beta=0$.

${ }^{2}$ It can be shown that this manifold is homeomorphic to $T S^{2}$, the tangent bundle on the sphere.
}

Thus, we have three primary constraints, corresponding to the three components of $\vec{\Phi} \equiv \vec{\Pi}_{P}=0$. The augmented Hamiltonian will therefore require three Lagrange multipliers, which we will assemble into a vector $\vec{u}$; this allows us to write the augmented Hamiltonian compactly as

$$
\begin{aligned}
\mathcal{H}_{A}= & \vec{\Pi}_{Q} \cdot \dot{\vec{Q}}-\mathcal{L}+\vec{u} \cdot \vec{\Pi}_{P} \\
= & \frac{1}{2} \vec{\Pi}_{Q}^{2}+\vec{\Pi}_{Q} \cdot(\vec{\nabla} \times \vec{P})+\frac{1}{2}(\vec{\nabla} \cdot \vec{Q})^{2} \\
& +V(X, Y)+\vec{u} \cdot \vec{\Pi}_{P} .
\end{aligned}
$$

We must now see whether the primary constraints $\vec{\Phi}=0$ are closed under the time evolution of the system, thereby obtaining secondary constraints and/or values for the Lagrange multipliers $\vec{u}$. The preservation of the primary constraints leads to a set of secondary constraints $\vec{\Psi}$ :

$$
\begin{aligned}
0 & =\dot{\vec{\Phi}}=\left\{\vec{\Pi}_{P}, H_{A}\right\} \\
& =\vec{\nabla} \times \vec{\Pi}_{Q}-\frac{\partial V}{\partial \vec{P}} \equiv \vec{\Psi} .
\end{aligned}
$$

However, preservation of the secondary constraints leads to an equation involving the unknown Lagrange multipliers $\vec{u}$ :

$$
\begin{aligned}
0= & \dot{\Psi}_{i}=\left\{\left(\vec{\nabla} \times \vec{\Pi}_{Q}\right)_{i}-\frac{\partial V}{\partial P_{i}}, H_{A}\right\} \\
= & -\left[\vec{\nabla} \times\left(\frac{\partial V}{\partial \vec{Q}}\right)\right]_{i}-\frac{\partial^{2} V}{\partial P_{i} \partial P_{j}} u_{j} \\
& -\frac{\partial^{2} V}{\partial P_{i} \partial Q_{j}}\left[\vec{\Pi}_{Q}+\vec{\nabla} \times \vec{P}\right]_{j} .
\end{aligned}
$$

If we define a vector $\vec{v}$ as

$$
v_{i} \equiv\left[\vec{\nabla} \times\left(\frac{\partial V}{\partial \vec{Q}}\right)\right]_{i}+\frac{\partial^{2} V}{\partial P_{i} \partial Q_{j}}\left[\vec{\Pi}_{Q}+\vec{\nabla} \times \vec{P}\right]_{j}
$$

and a matrix $\mathcal{M}_{i j}$ as

$$
\mathcal{M}_{i j}=\frac{\partial^{2} V}{\partial P_{i} \partial P_{j}},
$$

then Eq. (22) reduces to the equation

$$
\mathcal{M}_{i j} u_{j}+v_{i}=0 \text {. }
$$

This equation will determine some or all of the components of $\vec{u}$; the number of components so determined is equal to the rank of the matrix $\mathcal{M}$.

What remains is to find an expression for $\mathcal{M}_{i j}$. Using the chain rule, it is not hard to show that 
$\mathcal{M}_{i j}=-4 V_{X} \delta_{i j}+16 \kappa_{1} P_{i} P_{j}+32 \kappa_{2} P_{(i} Q_{j)}+16 \kappa_{3} Q_{i} Q_{j}$,

where $V_{X}=\partial V / \partial X=\kappa_{1} X+\kappa_{2} Y+\lambda_{1}$. To solve (25), we need to invert $\mathcal{M}_{i j}$. By taking an ansatz of the form

$$
\left(\mathcal{M}^{-1}\right)_{j k}=\mathcal{A} \delta_{j k}+\mathcal{B} P_{j} P_{k}+2 \mathcal{C} P_{(j} Q_{k)}+\mathcal{D} Q_{j} Q_{k}
$$

and requiring that $\mathcal{M}_{i j}\left(\mathcal{M}^{-1}\right)_{j k}=\delta_{i k}$, we find that the inverse exists for a generic point in field space, with

$$
\begin{aligned}
& \mathcal{A}=-\frac{1}{4 V_{X}} \\
& \mathcal{B}=\frac{1}{V_{X} \mathcal{Q}}\left[-\kappa_{1} V_{X}+4 \vec{Q}^{2}\left(\kappa_{1} \kappa_{3}-\kappa_{2}^{2}\right)\right] \\
& \mathcal{C}=\frac{1}{V_{X} \mathcal{Q}}\left[-\kappa_{2} V_{X}-4(\vec{P} \cdot \vec{Q})\left(\kappa_{1} \kappa_{3}-\kappa_{2}^{2}\right)\right] \\
& \mathcal{D}=\frac{1}{V_{X} \mathcal{Q}}\left[-\kappa_{3} V_{X}+4 \vec{P}^{2}\left(\kappa_{1} \kappa_{3}-\kappa_{2}^{2}\right)\right],
\end{aligned}
$$

where

$$
\begin{aligned}
\mathcal{Q} \equiv & V_{X}^{2}-4 V_{X}\left(\kappa_{1} \vec{P}^{2}+2 \kappa_{2} \vec{P} \cdot \vec{Q}+\kappa_{3} \vec{Q}^{2}\right) \\
& +16\left(\kappa_{1} \kappa_{3}-\kappa_{2}^{2}\right)\left[\vec{P}^{2} \vec{Q}^{2}-(\vec{P} \cdot \vec{Q})^{2}\right]
\end{aligned}
$$

At a generic point in field space, this is well defined, and so we can invert (25) to determine the three Lagrange multipliers $\vec{u}$ in terms of the other fields. The overall Hamiltonian density for the system would then be

$$
\begin{aligned}
\mathcal{H}_{A}= & \frac{1}{2} \vec{\Pi}_{Q}^{2}+\vec{\Pi}_{Q} \cdot(\vec{\nabla} \times \vec{P})+\frac{1}{2}(\vec{\nabla} \cdot \vec{Q})^{2}+V(X, Y) \\
& -\left(\Pi_{P}\right)_{i}\left(\mathcal{M}^{-1}\right)_{i j} v_{j} .
\end{aligned}
$$

Further, if this inverse is well defined, we can count the number of d.o.f. of the theory. We have six fields $(\vec{P}$ and $\vec{Q})$, three primary constraints $\vec{\Pi}_{P}=0$, and three secondary constraints given in (21). Thus, the number of d.o.f. for a general point in field space is

$$
N_{\text {dof }}=6-\frac{1}{2}(3+3+0)=3 .
$$

It is evident, however, that the inverse matrix (27) is not well defined when either $\mathcal{Q}$ or $V_{X}$ vanishes. This presents a dilemma. If we start with an initial-data configuration satisfying the constraints and for which $\mathcal{Q}$ and $V_{X}$ are nonvanishing, then the Hamiltonian (28) becomes singular if the fields ever evolve to a point where $V_{X}$ or $\mathcal{Q}$ vanishes. Alternately, one could construct a Hamiltonian under the assumption that $V_{X}$ and/or $\mathcal{Q}$ vanish. In this case, the matrix
$\mathcal{M}_{i j}$ would not be of full rank. This would leave one or more components of $\vec{u}$ undetermined in (25); it would also require that certain components of $\vec{v}$ (those not in the range of $\mathcal{M}_{i j}$ ) vanish automatically, leading to additional constraints. The iterative constraint-generation procedure described in Sec. II would therefore have to continue; assuming that it did not lead to an inconsistency, the resulting theory would necessarily have fewer d.o.f. than the theory constructed for a generic point in field space.

This "loss" of a d.o.f. at certain points in field space was noted in Ref. [12] in the context of a vector field model with an unorthodox kinetic term. It has also been noted in certain vector field models in curved spacetime $[9,13]$. While these features of those other models are troubling, one could perhaps argue that the singularities of those models occur at nongeneric points in field space that in some sense are well separated from "typical" field configurations. In that sense, those models might still be viable.

What makes the singularity in the present case especially vexing, however, is that we cannot make such an argument. The Hamiltonian is singular when $V_{X}=0$, and by definition $V_{X}=0$ holds for all points in the vacuum manifold. The above arguments imply that the evolution between field configurations "on" the vacuum manifold and field configurations "off" the vacuum manifold is rather ill posed, since Hamilton's equation for $\vec{P}$ is

$$
\frac{d P_{i}}{d t}=\frac{\delta H_{A}}{\delta \Pi_{P i}}=-\left(\mathcal{M}^{-1}\right)_{i j} v_{j}
$$

This casts serious doubt on the viability of such a field theory as a candidate for dynamical Lorentz symmetry violation. The field configurations with $\vec{v} \neq 0$ are generic in field space, both on and off the vacuum manifold. Most small perturbations away from the vacuum manifold would therefore have $\vec{v} \neq 0$ as they evolve "back towards" the vacuum manifold. But since $\mathcal{M}^{-1}$ becomes singular as the fields approach the vacuum manifold, we are forced to conclude that $d \vec{P} / d t$ will diverge as the fields evolve back towards the vacuum manifold.

This statement may seem to be at odds with the work of Altschul et al. [4]. In that work, the authors linearized the equations of motion (13) about a constant background tensor for which $V_{X}=V_{Y}=0$. The authors allowed for the presence of "massive modes," field configurations for which $V \neq 0$. However, their results showed that at linear order the field invariants $X$ and $Y$ (and thus the value of $V$ itself) are constant in time. In their terminology, the massive modes are "nonpropagating." Altschul et al. also raised the question of whether these modes might become "propagating modes" at higher orders in perturbation theory. My present work answers this question in the affirmative; in fact, it is the evolution of the field from $V \neq 0$ to $V=0$ that leads to the pathology I have found. 
In constructing linearized versions of a nonlinear field theory, it is often implicitly assumed that the solutions to the linearized equations have a one-to-one correspondence with "small solutions" of the full nonlinear equations of motion. A theory for which this correspondence can be drawn is said to be linearization stable [14]. However, not all models have this property; in many models, one can find solutions to the linearized equations that do not correspond to small solutions of the full nonlinear equations. Showing whether a given set of nonlinear differential equations is linearization stable is a complex question, and a full discussion would be beyond the scope of this paper. However, the fact that solutions with $V \neq 0$ remain small in the linearized theory [4], but can diverge in the nonlinear theory, would suggest that the antisymmetric tensor evolution equations (13) are not in fact linearization stable.

More recently, Hernaski has also examined the consequences of spontaneous Lorentz symmetry violation in the context of an antisymmetric rank-2 tensor [15]. That work used general symmetry considerations to find the most general form of an effective Lagrangian for the NambuGoldstone modes arising from this sort of spontaneous Lorentz symmetry breaking. While this construction does not involve the problematic massive modes of the model, the fact that the "low-energy limit" of an action (11) is so ill posed means that it is unclear if Hernaski's effective Lagrangian could actually correspond to the low-energy limit of a model involving a fundamental tensor field breaking Lorentz symmetry. However, in the construction, Hernaski remained agnostic about the mechanism by which this vacuum expectation value arose, and other mechanisms could possibly still give rise to such an effective Lagrangian (see Sec. V).

\section{GENERAL FIELD THEORIES}

\section{A. Invariants and constraints}

Confronted with this problem, two natural questions arise: why does this pathology occur, and does it affect other tensor field models? A similar pathology was noted in Ref. [12] for the " $V$-field," a model consisting of a vector field $A_{a}$ governed by the action

$$
S=\int d^{4} x\left[\nabla_{a} A^{b} \nabla_{b} A^{a}-V\left(A_{a}\right)\right],
$$

where $V\left(A^{a}\right)=\kappa\left(A^{a} A_{a}-b\right)^{2}$. This Lagrangian can be integrated by parts to cast it in the alternate form

$$
S=\int d^{4} x\left[\left(\nabla_{a} A^{a}\right)^{2}-V\left(A_{a}\right)\right] .
$$

In this form, it is evident that the model has three constraints, since the velocities of the spatial components
$\dot{\vec{A}}$ do not appear. However, the $3 \times 3$ matrix $\mathcal{M}_{i j}$ from that work [defined analogously to (24) here] is

$$
\mathcal{M}_{i j}=\frac{\partial^{2} V}{\partial A_{i} \partial A_{j}}=4 \kappa\left[\delta_{i j}\left(A_{a} A^{a}-b\right)+2 A_{i} A_{j}\right] .
$$

This can be seen to have a rank of 1 if $A_{a} A^{a}=b$ and 3 otherwise. Since $\mathcal{M}$ has full rank off the vacuum manifold but has a nontrivial null space on the vacuum manifold, the inverse for $\mathcal{M}$ becomes singular on the vacuum manifold, leading to the same pathology we found in the antisymmetric tensor case.

In the other two models discussed in Ref. [12], however, the number of constraints is smaller. For a general kinetic term of the form

$$
\mathcal{L}_{K}=c_{1}\left(\nabla_{a} A_{b}\right)\left(\nabla^{a} A^{b}\right)+c_{3}\left(\nabla_{a} A_{b}\right)\left(\nabla^{b} A^{a}\right)
$$

and the same potential $V\left(A_{a}\right)$, there is one primary constraint if $c_{1}=-c_{3}$ (this is the familiar Maxwell kinetic term) and no primary constraints if $c_{1} \neq-c_{3}$ and $c_{1} \neq 0$.

The pathology therefore seems to depend on the number of primary constraints in the model. Specifically, both the $V$-field model and the antisymmetric tensor model have the property that on the vacuum manifold the rank of the matrix $\mathcal{M}$ is less than the number of primary constraints. In both cases, the matrix $\mathcal{M}$ is constructed by taking the second derivatives of the potential $V$ with respect to the "constrained fields": $\vec{P}$ for the antisymmetric tensor and $\vec{A}$ for the $V$-field. It is the failure of this matrix to be full rank on the vacuum manifold that leads to a singular Hamiltonian.

It is not hard to show that the rank of any matrix constructed in such a way is bounded above by the number of invariants used to construct the potential $V$. Suppose we have a potential $V\left(X_{1}, X_{2}, \ldots, X_{N}\right)$, where the quantities $X_{A}$ are in turn functions of some subset of the field variables $\psi_{\alpha}=\left\{\psi_{1}, \psi_{2}, \ldots, \psi_{n}\right\}$, with $n>N$. The analogous $n \times n$ matrix will then be

$$
\mathcal{M}_{\alpha \beta}=\frac{\partial^{2} V}{\partial \psi_{\alpha} \partial \psi_{\beta}} .
$$

If we imagine diagonalizing this matrix, we can see that the null space of this matrix corresponds to the "directions" in field space in which the potential is flat. More explicitly, we can use the chain rule to rewrite $\mathcal{M}_{\alpha \beta}$ as

$$
\mathcal{M}_{\alpha \beta}=\frac{\partial^{2} V}{\partial X_{A} \partial X_{B}} \frac{\partial X_{A}}{\partial \psi_{\alpha}} \frac{\partial X_{B}}{\partial \psi_{\beta}}+\frac{\partial V}{\partial X_{A}} \frac{\partial^{2} X_{A}}{\partial \psi_{\alpha} \partial \psi_{\beta}},
$$

where a summation over $A$ and $B$ is understood. In the vacuum manifold, the second term in (36) will vanish (since the vacuum manifold, by definition, extremizes $V$ with respect to of all its arguments.) The first term, 
meanwhile, will have a rank of at most $N$, the number of invariants used to construct $V$. This implies that in the vacuum manifold the rank of $\mathcal{M}_{\alpha \beta}$ will be less than $n$, the dimension of the space it is defined on. ${ }^{3}$

Effectively, this means that if the potential has "too many flat directions" we risk the rank of this matrix being too small on the vacuum manifold. ${ }^{4}$ But for a given tensor field, there are only a limited number of independent Lorentz invariants that can be constructed from it, and this number of invariants places an upper bound on the number of "nonflat" directions that $V$ can have. It is therefore possible that a given tensor field may not have enough invariants to reduce the nullity (and increase the rank) of $\mathcal{M}_{\alpha \beta}$ sufficiently.

This illustrates why the matrix $\mathcal{M}$ is not of full rank in either the antisymmetric tensor model or the $V$-field model. In both cases, we are constructing the matrix $\mathcal{M}$ by taking the derivatives of $V$ with respect to three constrained fields: the "electric vector" $\vec{P}$ for the antisymmetric tensor or the spatial components of $A_{a}$ for the $V$-field. But there are only two invariants that can be constructed out of an antisymmetric tensor $B_{a b}$ and only one that can be constructed out of a vector field $A_{a}$, and so the rank of $\mathcal{M}$ decreases when we are on the vacuum manifold.

\section{B. Constraint structure}

Given the above features of the antisymmetric tensor and $V$-field models, one might conjecture that any model which has "more constraints than invariants" would exhibit a similar pathology. However, the picture is not so simple. In particular, the structure of the constraints was critical to the argument; the problematic conditions followed from the preservation of the secondary constraints, and the preservation of the primary constraints did not determine any of the Lagrange multipliers $u_{\alpha}$ in any way. In this section, I will therefore proceed through the constraint algebra for a more general field theory to see which features of these models led to this pathology and whether this simple picture of the pathology arising from more constraints than invariants might hold under more general circumstances.

Suppose we consider a field theory in terms of some set of fields $\psi_{\alpha}(\alpha=1, \ldots, n)$ for which the dynamics are given by a Lagrangian that is quadratic in these fields' derivatives, both spatial and temporal. Suppose, further, that the "kinetic terms" $\mathcal{L}_{K}$ of the Lagrangian depend only on these derivatives, so we have

\footnotetext{
${ }^{3}$ In both of the explicit models under consideration, the second term in (36) is of rank $n$ when $V_{X} \neq 0$; in fact, it works out to be proportional to $\delta_{\alpha \beta}$. This may not occur in a more general case.

${ }^{4}$ Note that if we consider all of the fields $\psi_{\alpha}$, rather than just a subset, the nullity of $\mathcal{M}_{\alpha \beta}$ is precisely the dimension of the vacuum manifold in field space, and the rank of $\mathcal{M}_{\alpha \beta}$ is its codimension in field space.
}

$\mathcal{L}_{K}=\frac{1}{2} \sum_{\alpha, \beta \leq n}\left[\mathcal{P}_{\alpha \beta} \dot{\psi}_{\alpha} \dot{\psi}_{\beta}+2 \mathcal{Q}_{\alpha i \beta} \dot{\psi}_{\alpha} \nabla_{i} \psi_{\beta}+\mathcal{R}_{i \alpha j \beta} \nabla_{i} \psi_{\alpha} \nabla_{j} \psi_{\beta}\right]$

The quantities $\mathcal{P}_{\alpha \beta}, \mathcal{Q}_{\alpha i \beta}$, and $\mathcal{R}_{i \alpha j \beta}$ are assumed to be numerical coefficients, independent of the fields and of spacetime coordinates. Here and in what follows, we will need to explicitly write out the summations over field indices; repeated indices should not be assumed to be summed if the summation is not stated explicitly. However, the summations over the spatial indices $i$ and $j$ will remain implicit.

Via various field and coefficient redefinitions, it can be shown (see Appendix) that a set of kinetic terms of this form can always be rewritten in the form

$$
\begin{aligned}
\mathcal{L}_{K}= & \frac{1}{2} \sum_{\alpha, \beta \leq m} \mathcal{P}_{\alpha \beta}\left(\dot{\psi}_{\alpha}+\sum_{\gamma \leq n} \mathcal{S}_{\alpha i \gamma} \nabla_{i} \psi_{\gamma}\right)\left(\dot{\psi}_{\beta}+\sum_{\delta \leq n} \mathcal{S}_{\beta i \delta} \nabla_{i} \psi_{\delta}\right) \\
& +\sum_{\alpha, \beta>m} \mathcal{Q}_{\alpha i \beta} \dot{\psi}_{\alpha} \nabla_{i} \psi_{\beta}+\frac{1}{2} \sum_{\alpha, \beta \leq n} \mathcal{R}_{i \alpha j \beta} \nabla_{i} \psi_{\alpha} \nabla_{j} \psi_{\beta}
\end{aligned}
$$

where $\mathcal{P}_{\alpha \beta}$ is a nondegenerate diagonal matrix and $m \leq n$. The advantage of this form is that it is particularly simple to identify the primary constraints. For $\alpha \leq m$, we have

$$
\pi_{\alpha} \equiv \frac{\partial \mathcal{L}_{K}}{\partial \dot{\psi}_{\alpha}}=\mathcal{P}_{\alpha \alpha}\left(\dot{\psi}_{\alpha}+\sum_{\delta \leq n} \mathcal{S}_{\alpha i \delta} \nabla_{i} \psi_{\delta}\right)
$$

which can be easily inverted to find the velocities in terms of the momenta and derivatives. For $\alpha>m$, meanwhile, we have

$$
\pi_{\alpha}=\sum_{\beta>m} \mathcal{Q}_{\alpha i \beta} \nabla_{i} \psi_{\beta}
$$

which can easily be seen to be a constraint equation:

$$
\Phi_{\alpha} \equiv \pi_{\alpha}-\sum_{\beta>m} \mathcal{Q}_{\alpha i \beta} \nabla_{i} \psi_{\beta}=0
$$

The number of primary constraints in this model is therefore $n-m$.

Let us now suppose that the full Lagrangian density of the model is of the form

$$
\mathcal{L}=\mathcal{L}_{K}-V\left(\psi_{\alpha}\right)
$$

with $\mathcal{L}_{K}$ of the form given in (38) and $V\left(\psi_{\alpha}\right)$ a potential that does not depend on any field derivatives. Then, the base Hamiltonian density of this model will be 


$$
\begin{aligned}
\mathcal{H}_{0}= & \frac{1}{2} \sum_{\alpha, \beta \leq m} \mathcal{P}_{\alpha \beta}^{-1} \pi_{\alpha} \pi_{\beta}-\sum_{\alpha \leq m} \sum_{\beta \leq n} \pi_{\alpha} \mathcal{S}_{\alpha i \beta} \nabla_{i} \psi_{\beta} \\
& -\frac{1}{2} \sum_{\alpha, \beta \leq n} \mathcal{R}_{i \alpha j \beta} \nabla_{i} \psi_{\alpha} \nabla_{j} \psi_{\beta}+V\left(\psi_{\alpha}\right) .
\end{aligned}
$$

The augmented Hamiltonian density can then be obtained by adding a set of Lagrange multiplier terms,

$$
\mathcal{H}_{L M}=\sum_{\alpha>m} u_{\alpha}\left(\pi_{\alpha}-\sum_{\beta>m} \mathcal{Q}_{\alpha i \beta} \nabla_{i} \psi_{\beta}\right),
$$

to $(43)$.

The first requirement we will need to impose to reproduce the pathology found in the previous section is to require that the coefficients $\mathcal{Q}_{\alpha i \beta}$ in (38) vanish for $\alpha, \beta>m$. To see this, note that the time evolution of a primary constraint $\Phi_{\alpha}$ will be given by

$$
\dot{\Phi}_{\alpha}=\left\{\Phi_{\alpha}, H_{0}\right\}+\left\{\Phi_{\alpha}, H_{L M}\right\},
$$

where $H_{0} \equiv \int d^{3} x \mathcal{H}_{0}$ and $H_{L M} \equiv \int d^{3} x \mathcal{H}_{L M}$. The latter Poisson bracket can be evaluated to be

$$
\left\{\Phi_{\alpha}, H_{L M}\right\}=-\sum_{\beta>m} \mathcal{Q}_{\alpha i \beta} \nabla_{i} u_{\beta} .
$$

As noted above, the pathology in the previous section arises from the preservation of the secondary constraints, not the primary constraints. If the Lagrange multipliers enter at this stage, then the preservation of the primary constraints will at least partially determine them, and the chain of logic will diverge at this stage. Thus, for a model to follow the same logical chain, we must have these terms vanishing for all $\alpha$, and so we must have $\mathcal{Q}_{\alpha i \beta}=0$. In the language of Dirac, this means that the primary constraints are all first class, since they all mutually commute with each other. Both the antisymmetric tensor model and the $V$-field model have this property.

Making this assumption, the secondary constraints $\Psi_{\alpha} \equiv\left\{\Phi_{\alpha}, H_{0}\right\}$ can be calculated to be

$$
\Psi_{\alpha}=-\frac{\partial V}{\partial \psi_{\alpha}}-\sum_{\beta \leq n}\left(\mathcal{S}_{\alpha i \beta} \nabla_{i} \pi_{\beta}+\mathcal{R}_{i \alpha j \beta} \nabla_{i} \nabla_{j} \psi_{\beta}\right) .
$$

These secondary constraints will in turn need to be preserved, i.e., $\dot{\Psi}_{\alpha}=\left\{\Psi_{\alpha}, H_{A}\right\}=0$. In the pathological cases described above, the Lagrange multipliers entered into the analogous equation. To see where they enter here, we can calculate the Poisson bracket $\left\{\Phi_{\alpha}, H_{A}\right\}$; we obtain

$$
\dot{\Psi}_{\alpha}=v_{\alpha}-\sum_{\beta>m}\left(\frac{\partial^{2} V}{\partial \psi_{\alpha} \partial \psi_{\beta}} u_{\beta}+\mathcal{R}_{i \alpha j \beta} \nabla_{i} \nabla_{j} u_{\beta}\right),
$$

where $v_{\alpha}$ represents all terms that do not depend on the Lagrange multipliers $u_{\alpha}$.

The first term in the parentheses in (48) is the one that caused the pathology in the cases of the antisymmetric tensor and the $V$-field; the rank of the matrix

$$
\frac{\partial^{2} V}{\partial \psi_{\alpha} \partial \psi_{\beta}}=\mathcal{M}_{\alpha \beta}
$$

was different on the vacuum manifold than on a general point in field space. However, we can see from the above that in a more general model the derivative terms in (48) can also help to determine the Lagrange multipliers; the number of Lagrange multipliers that are determined by requiring that (48) vanishes is not necessarily just the rank of $\mathcal{M}_{\alpha \beta}$. For a model to have the vacuum manifold pathology described in the previous sections, it is sufficient for these derivative terms to vanish; in other words, $\mathcal{R}_{i \alpha j \beta}=0$ for $\alpha, \beta>m^{5}$

In summary, any Lagrangian of which the kinetic terms are of the form (38) (or can be put into this form) will suffer from the vacuum manifold pathology described above if the coefficients $\mathcal{Q}_{\alpha i \beta}=0$ and $\mathcal{R}_{i \alpha j \beta}=0$ when both $\alpha$ and $\beta$ are greater than $m$ and when the potential $V$ is constructed from fewer than $n-m$-field quantities depending on the fields $\psi_{\alpha}$ (with $\alpha>m$ ). Both the antisymmetric tensor model and the $V$-field model satisfy these criteria. The kinetic term (15) is of the form (38); a term corresponding to the $\mathcal{R}_{i \alpha j \beta}$ term does exist in the kinetic terms [specifically, the term $(\vec{\nabla} \cdot \vec{Q})^{2}$ ], but it only involves the "unconstrained" fields $\vec{Q}$ and not the constrained fields $\vec{P}$. Similarly, the kinetic term for the $V$-field Lagrangian (32), when decomposed into its time and space components, is

$$
\mathcal{L}_{K}=\left(\dot{A}_{0}-\vec{\nabla} \cdot \vec{A}\right)^{2},
$$

which does not contain any terms corresponding to the second or third summations in (38) at all.

The above-listed conditions appear to be sufficient for this pathology, but they may not be necessary. It is entirely possible that a model for which some of the primary constraints were second class, or for which the constrained fields appeared with spatial derivatives, could still have a Hamiltonian which became singular on the vacuum manifold. However, in either case, the Lagrange multipliers could not be solved for algebraically, and so the analysis of the Hamiltonian would not be nearly as straightforward. It is also unclear whether any physically well-motivated models with these features exist.

\footnotetext{
${ }^{5}$ Note that we can take $\mathcal{R}_{i \alpha j \beta}$ to be symmetric under the exchange of $i$ and $j$, since $\mathcal{R}_{i \alpha j \beta} \nabla_{i} \psi_{\alpha} \nabla_{j} \psi_{\beta}=\mathcal{R}_{i \alpha j \beta} \nabla_{j} \psi_{\alpha} \nabla_{i} \psi_{\beta}$ up to total derivatives.
} 


\section{DISCUSSION}

We have shown that a model which spontaneously breaks Lorentz symmetry may, unless constructed with care, have pathological evolution that is not immediately evident at the level of the Lagrangian. It is important to note that this technique is qualitatively different from much of the previous literature. Many investigations into the viability of such models have focused on their phenomenology, investigating the stability of the new fields (e.g., Ref. [16]) or investigating their interactions with matter (e.g., Ref. [17]). A framework applicable to some such models in a gravitational context was also put forward in Ref. [18]. Such phenomenological studies usually lead to a set of constraints on the parameters of the model. However, in general, these phenomenological constraints, no matter how stringent, will leave behind a small region of viable parameter space and cannot rule out a "fine-tuned" version of a model. The methods of the present work, in contrast, have the potential to completely rule out a model; the pathological behavior of the antisymmetric tensor model in Sec. III exists regardless of the values of the parameters $\left\{\kappa_{1}, \kappa_{2}, \kappa_{3}, \lambda_{1}, \lambda_{2}\right\}$ of the model.

This result has serious implications for the construction of such models. If we wish to build a model with a Lorentzviolating field, how can we reliably evade these pathologies? Assuming that the model under consideration can be written in the form (38), with $\mathcal{Q}_{\alpha i \beta}=0$ and $\mathcal{R}_{i \alpha j \beta}=0$ when $\alpha, \beta>m$, then the only way to avoid the pathology is to ensure that the rank of the matrix $\mathcal{M}_{\alpha \beta}$ is sufficiently high. This may not always be possible. For example, for the case of an antisymmetric 2-tensor $B_{a b}$, there are only two independent Lorentz invariants that can be constructed from it, namely $X$ and $Y$ as defined in (9). Since the kinetic term used in (11) gives rise to three constraints, it is simply not possible to write down a potential for $B_{a b}$ that does not give rise to pathological evolution. One could possibly change the kinetic term so that the model had no more than two primary constraints; however, this could very well give rise to instabilities, as discussed in the Introduction.

For tensor fields of higher rank or different symmetry type, a similar set of considerations would have to come into play. As an example, Kostelecký and Potting's linear cardinal gravity model [2] involves a symmetric rank-2 tensor field $C_{a b}$ in a flat background with a potential $V\left(C_{a b}\right)$ and the standard kinetic terms for a massless spin-2 field:

$$
\begin{aligned}
\mathcal{L}_{K}= & -\frac{1}{4}\left[\nabla_{c} C_{a b} \nabla^{c} C^{a b}-\nabla_{a} C \nabla^{a} C\right. \\
& \left.+2 \nabla_{a} C \nabla_{b} C^{a b}-2 \nabla_{b} C^{a b} \nabla_{c} C_{a}{ }^{c}\right] .
\end{aligned}
$$

Performing a $3+1$ decomposition, we find that the time derivatives of $C_{00}$ and $C_{0 i}$ do not appear in this Lagrangian, and thus this model will have four primary constraints.
Moreover, there are no cross-couplings between the derivatives (either spatial or temporal) of $C_{00}$ and $C_{0 i}$; this means that the appropriate $\mathcal{Q}_{\alpha i \beta}$ and $\mathcal{R}_{i \alpha j \beta}$ coefficients vanish in order for the general result of Sec. IV B to hold.

Given these features of linear cardinal gravity, one might be concerned that it runs the risk of suffering from the same vacuum-manifold pathology as the antisymmetric rank-2 tensor field. However, there is one important distinction: while there are only two independent invariants one can construct from an antisymmetric tensor field ( $X$ and $Y$ ), there are four independent invariants that can be constructed from a symmetric rank-2 tensor field:

$$
\begin{aligned}
& X_{1}=C_{a}{ }^{a} \\
& X_{2}=C_{a}{ }^{b} C_{b}{ }^{a} \\
& X_{3}=C_{a}{ }^{b} C_{b}{ }^{c} C_{c}{ }^{a} \\
& X_{4}=C_{a}{ }^{b} C_{b}{ }^{c} C_{c}{ }^{d} C_{d}{ }^{a} .
\end{aligned}
$$

Thus, there appear to be "just enough" invariants for a cardinal gravity model to avoid the vacuum-manifold pathology discussed in this work, so long as the potential has nontrivial dependencies on all four of these invariants. It must be emphasized, however, that having no more constraints than invariants is a necessary, not sufficient, condition to obtain a nonsingular Hamiltonian that can be extended throughout all of field space; it is possible that other, more subtle pathologies occur in such a model.

In the face of these difficulties, it is important to note that there are other methods by which models can include "naturally nonzero" fields. One could, for example, postulate that $B_{a b}$ is not a fundamental field but is instead a function of some other fundamental field which gains a vacuum expectation value. For example, a recent work by Assunção et al. [19] introduced a model in which $B_{a b}$ is a spinor condensate. It is not immediately clear whether the present results could be generalized to such models.

Another method to avoid these difficulties would be to simply constrain the field to be nonzero via the use of a Lagrange multiplier $\lambda$,

$$
S=\int d^{4} x\left[-\frac{1}{12} F_{a b c} F^{a b c}-\lambda(X-b)\right],
$$

where $X$ is defined as in (9) and $b \neq 0$ is a constant. The equation of motion for $\lambda$ is then simply $X=b$, and thus the tensor field $B_{a b}$ would be nonzero "in vacuum.",

\footnotetext{
${ }^{6}$ It is worth noting here that Hernaski's effective low-energy Lagrangian [15] could equally well arise from a "fundamental" Lagrangian of this type, rather than from a Lagrangian involving a potential for the fundamental field $B_{a b}$.
} 
This method introduces a new field to the model as well as a new primary constraint; more importantly, it also changes the algebra of the primary constraints in important ways [12]. If we perform Dirac-Bergmann analysis on this Lagrangian, the augmented Hamiltonian density can be shown to be

$$
\begin{aligned}
\mathcal{H}_{A}= & \frac{1}{2} \vec{\Pi}_{Q}^{2}+\vec{\Pi}_{Q} \cdot(\vec{\nabla} \times \vec{P})+\frac{1}{2}(\vec{\nabla} \cdot \vec{Q})^{2}+\lambda(X-b) \\
& +\vec{u} \cdot \vec{\Pi}_{P}+u_{\lambda} \varpi .
\end{aligned}
$$

Here, $\varpi$ is the conjugate momentum to $\lambda$; it is identically zero, and so the equation $\varpi=0$ is enforced by a fourth Lagrange multiplier $u_{\lambda}$ (in addition to the three Lagrange multipliers $\vec{u}$ enforcing the constraint $\vec{\Pi}_{P}=0$.) If the primary constraints are to be preserved, their Poisson brackets with the augmented Hamiltonian $H_{A}$ must vanish. The secondary constraints are found to be

$$
\vec{\Psi} \equiv 4 \lambda \vec{P}-\vec{\nabla} \times \vec{\Pi}_{Q}
$$

and

$$
\Psi \equiv-(X-b) .
$$

These secondary constraints must in turn be preserved under time evolution; after some algebra, it can be shown that

$$
\dot{\vec{\Psi}}=4\left[u_{\lambda} \vec{P}+\lambda \vec{u}+\vec{\nabla} \times(\lambda \vec{Q})\right]
$$

and

$$
\dot{\Psi}=4\left[\vec{P} \cdot \vec{u}-4 \vec{Q} \cdot\left(\vec{\Pi}_{Q}+\vec{\nabla} \times \vec{P}\right)\right] .
$$

These equations uniquely determine the Lagrange multipliers $\vec{u}$ and $u_{\lambda}$, so long as $\vec{P} \neq 0$ and $\lambda \neq 0$ :

$$
\begin{aligned}
u_{\lambda} & =-\frac{1}{P^{2}}\left[\vec{P} \cdot \vec{\nabla} \times(\lambda \vec{Q})+\lambda \vec{Q} \cdot\left(\vec{\Pi}_{Q}+\vec{\nabla} \times \vec{P}\right)\right] \\
\vec{u} & =-\frac{1}{\lambda}\left[u_{\lambda} \vec{P}+\vec{\nabla} \times(\lambda \vec{Q})\right] .
\end{aligned}
$$

For generic points in the vacuum manifold, the Hamiltonian is nonsingular, and the vacuum manifold pathology does not arise. In some sense, this is not a surprise; the pathology in the potential model arises when the field evolves onto or off of the vacuum manifold, but the field is "stuck" on the vacuum manifold in the Lagrange-multiplier model. ${ }^{7}$

\footnotetext{
${ }^{7}$ The above derivation also gives us the number of d.o.f. of the model (53). We have seven fields in the original Hamiltonian (the components of $B_{a b}$ and the Lagrange multiplier $\lambda$ ), four primary constraints $\left(\vec{\Pi}_{P}=0\right.$ and $\left.\varpi=0\right)$, and four secondary constraints ( $\vec{\Psi}=0$ and $\Psi=0$.) There are therefore $7-\frac{1}{2}(8)=3$ d.o.f. for this model. Note that, as discussed at length in Ref. [12], adding a Lagrange multiplier does not eliminate a d.o.f. from this model.
}

It must be said that some authors (myself included) find the use of Lagrange multipliers to be somewhat inelegant. This prejudice arises from classical particle mechanics, where a constrained model can often be viewed as a limit: one imagines a model where a potential energy is minimized on the constraint surface and then takes the limit of this model as the potential becomes infinitely strong. From this perspective, Lagrange multipliers are just an ad hoc approximation to a more fundamental theory. However, the situation is a lot more nuanced than that in classical field theory, particularly in the presence of models with primary constraints [12]. The present work shows that the use of Lagrange multipliers and/or composite fields may be unavoidable if one wants to model Lorentz symmetry violation with a tensor field, particularly one of higher rank, and that Lagrange-multiplier models may not be relatable to a "more fundamental" potential model at all.

Finally, one must remember that all of this analysis has been performed at the classical level. Ideally, one would want to take quantum effects into account as well. In the case of a set of scalar fields with a spontaneously broken internal symmetry, it is possible to take a fundamental quantum Lagrangian and calculate an effective potential for the expectation value of the field. The expectation value of the field will then obey a version of the classical equation of motion, with $V$ replaced by the effective potential, and so there is a well-defined parallel between classical models and quantum models of these fields. However, it is still an open question whether an effective potential formulation exists in the case of spontaneously broken Lorentz symmetry, and so it is unclear whether or not a similar correspondence between the classical and quantum pictures can be drawn. ${ }^{8}$ It may be that the pathologies found in the present work are an indication that such a correspondence does not actually exist for some fields undergoing spontaneously Lorentz symmetry breaking; more research on this subject is needed.

\section{ACKNOWLEDGMENTS}

The author would like to thank J. Tasson, B. Altschul, V. A. Kostelecký, Q. Bailey, and D. Garfisnkle for discussions during the preparation of this work. This research was supported in part by Perimeter Institute for Theoretical Physics. Research at Perimeter Institute is supported by the Government of Canada through Industry Canada and by the Province of Ontario through the Ministry of Economic Development \& Innovation.

\section{APPENDIX: CANONICAL FORM OF FIELD THEORY LAGRANGIAN}

Consider a Lagrangian for which the kinetic term is of the form (37) (reproduced here):

\footnotetext{
${ }^{8}$ I thank B. Altschul for this observation.
} 
$\mathcal{L}_{K}=\frac{1}{2} \sum_{\alpha, \beta \leq n}\left[\mathcal{P}_{\alpha \beta} \dot{\psi}_{\alpha} \dot{\psi}_{\beta}+2 \mathcal{Q}_{\alpha i \beta} \dot{\psi}_{\alpha} \nabla_{i} \psi_{\beta}+\mathcal{R}_{i \alpha j \beta} \nabla_{i} \psi_{\alpha} \nabla_{j} \psi_{\beta}\right]$.

In Sec. IV B, it was stated that a set of kinetic terms of this form can always be put into the form (38). This Appendix describes how this may be accomplished.

From the form of (37), it is fairly evident that we can take $\mathcal{P}_{\alpha \beta}$ to be symmetric under the exchange of $\alpha$ and $\beta$. Less evident, but equally important, is that $\mathcal{Q}_{\alpha i \beta}$ can also be taken to be symmetric under this exchange. The antisymmetric part of this array (when contracted with $\dot{\psi}_{\alpha} \nabla_{i} \psi_{\beta}$ ) can be expressed in terms of total derivatives:

$$
\begin{aligned}
& \left(\mathcal{Q}_{\alpha i \beta}-\mathcal{Q}_{\beta i \alpha}\right) \dot{\psi}_{\alpha} \nabla_{i} \psi_{\beta} \\
& \quad=\mathcal{Q}_{\alpha i \beta}\left(\dot{\psi}_{\alpha} \nabla_{i} \psi_{\beta}-\dot{\psi}_{\beta} \nabla_{i} \psi_{\alpha}\right) \\
& \quad=\nabla_{i}\left(\mathcal{Q}_{\alpha i \beta} \dot{\psi}_{\alpha} \psi_{\beta}\right)-\frac{\partial}{\partial t}\left(\mathcal{Q}_{\alpha i \beta}\left(\nabla_{i} \psi_{\alpha}\right) \psi_{\beta}\right) .
\end{aligned}
$$

With this in mind, the procedure for putting the kinetic terms in the form (38) is as follows:

(i) Since $\mathcal{P}_{\alpha \beta}$ can be taken to be real and symmetric, we can redefine the fields $\psi_{\alpha}$ (via an invertible linear transformation) so that the matrix $\mathcal{P}_{\alpha \beta}$ becomes diagonal. Moreover, we can reorder these fields so that $\mathcal{P}_{\alpha \alpha} \neq 0$ for all $\alpha \leq m$, and $\mathcal{P}_{\alpha \alpha}=0$ for $\alpha>m$. The kinetic terms of the Lagrangian then become

$$
\begin{aligned}
\mathcal{L}_{K}= & \frac{1}{2} \sum_{\alpha, \beta \leq m} \mathcal{P}_{\alpha \beta} \dot{\psi}_{\alpha} \dot{\psi}_{\beta}+\sum_{\alpha, \beta \leq n} \mathcal{Q}_{\alpha i \beta} \dot{\psi}_{\alpha} \nabla_{i} \psi_{\beta} \\
& +\frac{1}{2} \sum_{\alpha, \beta \leq n} \mathcal{R}_{i \alpha j \beta} \nabla_{i} \psi_{\alpha} \nabla_{j} \psi_{\beta},
\end{aligned}
$$

where the arrays $\mathcal{Q}_{\alpha i \beta}$ and $\mathcal{R}_{i \alpha j \beta}$ have been redefined after the field transformation.

(ii) By splitting the second sum in (A2) into three parts, integrating by parts, and applying the symmetry of

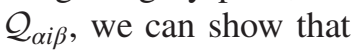

$$
\begin{aligned}
\sum_{\alpha, \beta \leq n} \mathcal{Q}_{\alpha i \beta} \dot{\psi}_{\alpha} \nabla_{i} \psi_{\beta} & \\
=\sum_{\alpha \leq m} \sum_{\beta \leq n} \mathcal{Q}_{\alpha i \beta} \dot{\psi}_{\alpha} \nabla_{i} \psi_{\beta} & +\sum_{\alpha \leq m} \sum_{\beta>m} \mathcal{Q}_{\alpha i \beta} \dot{\psi}_{\alpha} \nabla_{i} \psi_{\beta} \\
& +\sum_{m<\alpha, \beta \leq n} \mathcal{Q}_{\alpha i \beta} \dot{\psi}_{\alpha} \nabla_{i} \psi_{\beta} \\
= & \sum_{\alpha \leq m} \sum_{\beta \leq n} \tilde{\mathcal{Q}}_{\alpha i \beta} \dot{\psi}_{\alpha} \nabla_{i} \psi_{\beta}+\sum_{m<\alpha, \beta \leq n} \mathcal{Q}_{\alpha i \beta} \dot{\psi}_{\alpha} \nabla_{i} \psi_{\beta},
\end{aligned}
$$

where

$$
\tilde{\mathcal{Q}}_{\alpha i \beta}=\left\{\begin{array}{cc}
\mathcal{Q}_{\alpha i \beta} & \alpha \leq m, \beta \leq m \\
2 \mathcal{Q}_{\alpha i \beta} & \alpha \leq m, \beta>m
\end{array}\right.
$$

(iii) Since $\mathcal{P}_{\alpha \beta}$ is a diagonal, nondegenerate matrix for $\alpha, \beta \leq m$, it has an inverse. By then defining

$$
\mathcal{S}_{\alpha i \beta} \equiv \sum_{\gamma \leq m} \mathcal{P}_{\alpha \gamma}^{-1} \tilde{\mathcal{Q}}_{\gamma i \beta}
$$

we can then show that the first two summations in (A2) are equal to

$$
\begin{aligned}
\frac{1}{2} \sum_{\alpha, \beta \leq m} \mathcal{P}_{\alpha \beta} \dot{\psi}_{\alpha} \dot{\psi}_{\beta}+\sum_{\alpha, \beta \leq n} \mathcal{Q}_{\alpha i \beta} \dot{\psi}_{\alpha} \nabla_{i} \psi_{\beta}= & \frac{1}{2} \sum_{\alpha, \beta \leq m} \mathcal{P}_{\alpha \beta}\left(\dot{\psi}_{\alpha}+\sum_{\gamma \leq n} \mathcal{S}_{\alpha i \gamma} \nabla_{i} \psi_{\gamma}\right)\left(\dot{\psi}_{\beta}+\sum_{\delta \leq n} \mathcal{S}_{\beta i \delta} \nabla_{i} \psi_{\delta}\right) \\
& +\sum_{\alpha, \beta>m} \mathcal{Q}_{\alpha i \beta} \dot{\psi}_{\alpha} \nabla_{i} \psi_{\beta}-\frac{1}{2} \sum_{\alpha, \beta \leq m} \sum_{\delta, \gamma \leq n} \mathcal{P}_{\alpha \beta} \mathcal{S}_{\alpha i \gamma} \mathcal{S}_{\beta i \delta} \nabla_{i} \psi_{\gamma} \nabla_{j} \psi_{\delta}
\end{aligned}
$$

Effectively, what we have done here is simply "completed the square" of the first two summations in (A2).

(iv) The last sum in (A6) can then be absorbed into the last term in (A2), yielding a set of kinetic terms of the desired form:

$$
\mathcal{L}_{K}=\frac{1}{2} \sum_{\alpha, \beta \leq m} \mathcal{P}_{\alpha \beta}\left(\dot{\psi}_{\alpha}+\sum_{\gamma \leq n} \mathcal{S}_{\alpha i \gamma} \nabla_{i} \psi_{\gamma}\right)\left(\dot{\psi}_{\beta}+\sum_{\delta \leq n} \mathcal{S}_{\beta i \delta} \nabla_{i} \psi_{\delta}\right)+\sum_{\alpha, \beta>m} \mathcal{Q}_{\alpha i \beta} \dot{\psi}_{\alpha} \nabla_{i} \psi_{\beta}+\frac{1}{2} \sum_{\alpha, \beta \leq n} \mathcal{R}_{i \alpha j \beta} \nabla_{i} \psi_{\alpha} \nabla_{j} \psi_{\beta} .
$$


[1] V. A. Kostelecký, Gravity, Lorentz violation, and the standard model, Phys. Rev. D 69, 105009 (2004).

[2] V. A. Kostelecký and R. Potting, Gravity from spontaneous Lorentz violation, Phys. Rev. D 79, 065018 (2009).

[3] M. D. Seifert, Vector models of gravitational Lorentz symmetry breaking, Phys. Rev. D 79, 124012 (2009).

[4] B. Altschul, Q. G. Bailey, and V. A. Kostelecký, Lorentz violation with an antisymmetric tensor, Phys. Rev. D 81, 065028 (2010).

[5] T. Jacobson and D. Mattingly, Gravity with a dynamical preferred frame, Phys. Rev. D 64, 024028 (2001).

[6] J. Bekenstein, Relativistic gravitation theory for the modified Newtonian dynamics paradigm, Phys. Rev. D 70, 083509 (2004).

[7] J. W. Moffat, Scalar-tensor-vector gravity theory, J. Cosmol. Astropart. Phys. 03 (2006) 004.

[8] P. A. M. Dirac, Lectures on Quantum Mechanics (Yeshiva University, New York, 1964).

[9] J. Isenberg and J. Nester, The effect of gravitational interaction on classical fields: A hamilton-dirac analysis, Ann. Phys. (N.Y.) 107, 56 (1977).

[10] M. D. Seifert, Dynamical Lorentz symmetry breaking and topological defects, Phys. Rev. D 82, 125015 (2010).

[11] M. D. Seifert, Monopole Solution in a Lorentz-Violating Field Theory, Phys. Rev. Lett. 105, 201601 (2010).
[12] M. D. Seifert, Constraints and degrees of freedom in Lorentz-violating field theories, Phys. Rev. D 99, 045003 (2019).

[13] D. Garfinkle, J. Isenberg, and J. M. Martin-Garcia, Constraint equations in Einstein-aether theories and the weak gravitational field limit, Phys. Rev. D 86, 084009 (2012).

[14] J. E. Marsden and A. E. Fischer, Linearization stability of nonlinear partial differential equations, in Proceedings of Symposia in Pure Mathematics (AMS, 1975), p. 219.

[15] C. A. Hernaski, Spontaneous breaking of Lorentz symmetry with an antisymmetric tensor, Phys. Rev. D 94, 105004 (2016).

[16] M. D. Seifert, Stability of spherically symmetric solutions in modified theories of gravity, Phys. Rev. D 76, 064002 (2007).

[17] J. W. Elliott, G. D. Moore, and H. Stoica, Constraining the new aether: Gravitational Cherenkov radiation, J. High Energy Phys. 08 (2005) 066.

[18] Q. Bailey and V. A. Kostelecký, Signals for Lorentz violation in post-Newtonian gravity, Phys. Rev. D 74, 045001 (2006).

[19] J. F. Assunção, T. Mariz, J. R. Nascimento, and A. Yu Petrov, Dynamical Lorentz symmetry breaking in a tensor bumblebee model, arXiv:1902.10592. 